\title{
The anticancer effect of Huaier (Review)
}

\author{
XIAOJIN SONG, YAMING LI, HANWEN ZHANG and QIFENG YANG
}

Department of Breast Surgery, Qilu Hospital, Shandong University,

School of Medicine, Jinan, Shandong 250012, P.R. China

Received December 11, 2014; Accepted March 24, 2015

DOI: 10.3892/or.2015.3950

\begin{abstract}
Trametes robiniophila Murr. (Huaier) is a sandy beige mushroom found on the trunks of trees and has been widely used in traditional Chinese medicine (TCM) for $\sim 1,600$ years. The anticancer effects of Huaier have attracted increasing worldwide interest in recent years. Accumulating evidence suggests that the anticancer mechanism of Huaier may be associated with various biological activities, such as inhibition of cell proliferation, anti-metastasis, interference with tumor angiogenesis and tumor-specific immunomodulatory effect. Animal and experimental studies suggest that Huaier is a promising anticancer agent. Further clinical research is warranted to illustrate the untapped chemopreventive and therapeutic potential of Huaier either alone or in conjunction with existing therapies.
\end{abstract}

\section{Contents}

1. Introduction

2. Anti-proliferative effect of Huaier

3. Anti-metastatic effect of Huaier

4. Anti-angiogenic effect of Huaier

5. Apoptosis induction by Huaier

6. Inhibition of cancer stem cells (CSCs) by Huaier

7. Tumor-specific immunodulatory effect of Huaier

8. Therapeutic perspectives and conclusions

\section{Introduction}

Cancer is a major cause of morbidity and mortality worldwide and remains a formidable disease. According to the National Cancer Institute (NCI), it was estimated that there would be $1,665,540$ new cases of all cancer types and 585,720 people

Correspondence to: Professor Qifeng Yang, Department of Breast Surgery, Qilu Hospital, Shandong University, School of Medicine, 44 Wenhua Xi Road, Jinan, Shandong 250012, P.R. China E-mail: qifengy@gmail.com

Key words: Huaier, cancer, apoptosis, angiogenesis, metastasis, immunomodulatory, cancer stem cell would succumb to cancer in 2014. Conventional treatment for cancer patients include surgery, radiotherapy and chemotherapy. Over the last few decades, adjuvant chemotherapy following surgery has been proven to decrease recurrence and improve patient survival, yet may subsequently promote other malignancies such as ovarian (1), bowel (2), gastro-oesophageal (3) and pancreatic (4) cancers. Recently, several advanced alternative treatments including gene and targeted therapy are attracting worldwide attention $(5,6)$. However, these treatments are not effective, and resistance against chemotherapy, toxicity, side-effects and the unaffordable cost for most patients require the search for comparatively natural products or non-toxic drugs (7). Therefore, it is of urgent need in the biomedical science to develop novel anticancer agents with fewer and less debilitating side-effects and reduced drug resistance to satisfy the unfulfilled therapeutic demand of cancer patients.

Agents derived from natural sources exert a multitude of effects on chronic inflammation-driven diseases including cancer (8). Recently, much attention has been focused on medicinal plants or herbs as potential sources of new therapeutic anticancer drugs due to their lack of toxic effects, relatively lower cost and enhanced effectiveness (9-11). It was reported that these agents increase chemotherapeutic efficacy, reduce toxicity, and prolong the survival time, improve the immune functions and the quality of life of cancer patients (12).

Trametes robiniophila Murr. (Huaier) is a sandy beige mushroom found on the trunks of trees and was first recorded by Shi-Zhen Li, a famous Chinese practitioner in the Ming Dynasty. Huaier has been widely used in traditional Chinese medicine (TCM) for $\sim 1,600$ years (13). However, the anticancer effects of Huaier which have attracted increasing worldwide interest have only been researched in recent years (14-16). Accumulating evidence suggests that the anticancer mechanisms of Huaier effects may be associated with various biological activities, such as inhibition of cell proliferation (15), anti-metastasis (17), interference with tumor angiogenesis (14) and tumor-specific immunomodulatory effect $(16,18)$. Huaier was found to be a putative anticancer agent with broad therapeutic value following elaborate molecular studies conducted in the recent past. Irrespective of its effectiveness as a complementary therapy for cancer, to date, there is no systematic review available concerning the anticancer effects of Huaier and the underlying mechanisms. Understanding the mechanisms involved in the anticancer action of Huaier should provide useful information for its possible application in clinical cancer therapy and also in cancer prevention. This 


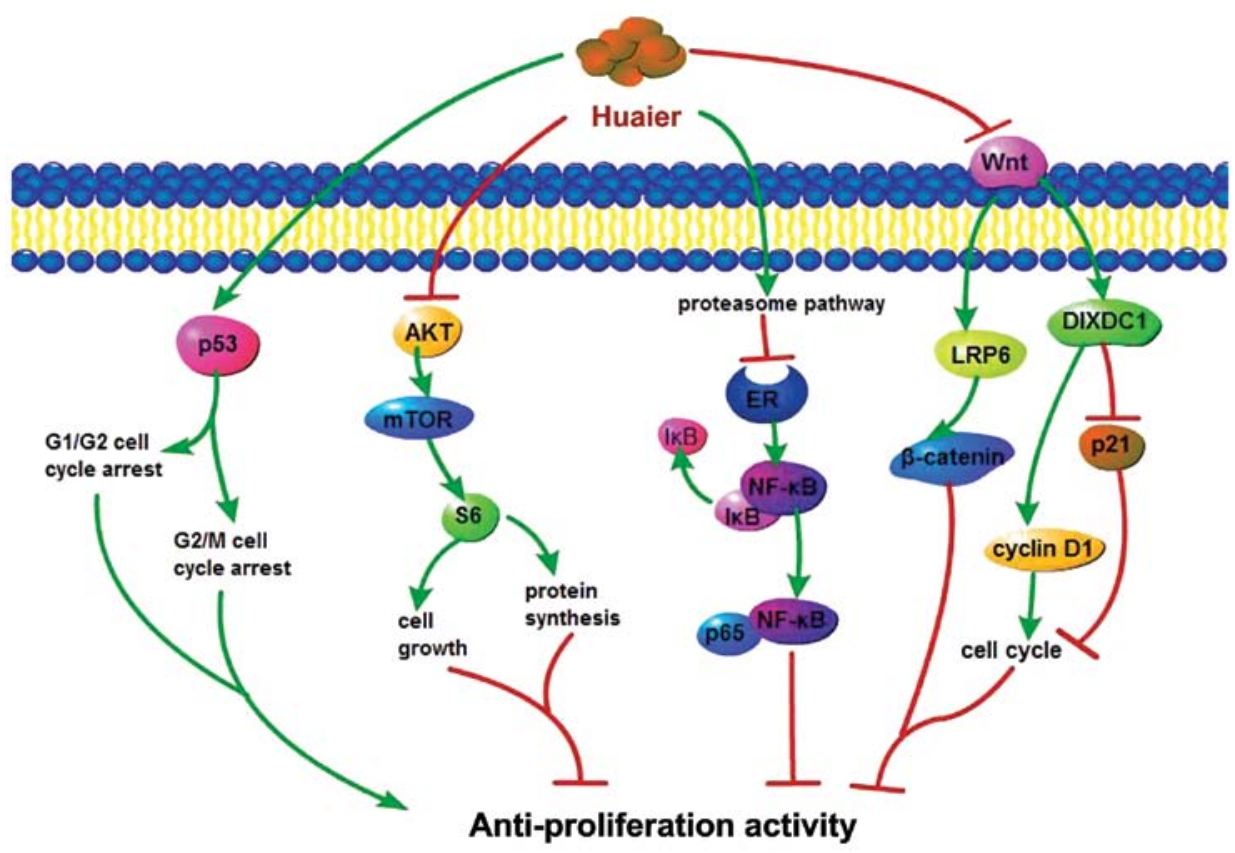

Figure 1. Huaier inhibits cancer cell proliferation via several classic pathways. Enhanced p53 expression inhibits cell proliferation via p53-induced cell-cycle arrest in MCF-7, MDA-MB-231 and A875 cells. The downregulation of pAKT/mTOR/S6 kinase pathway in ovarian epithelial cancer cells is also closely associated with this process. Huaier extract abolishes the effect of estrogen on the activation of NF- $\kappa \mathrm{B}$ and thus suppresses the proliferation of breast cancer cells induced by estrogen. In addition, inhibition of the Wnt/ $\beta$-catenin pathway is involved in the anti-proliferative effects of Huaier.

review briefly summarizes the literature published to date reporting the anticancer effect of Huaier and its underlying mechanisms.

\section{Anti-proliferative effect of Huaier}

Cell proliferation is an indispensible process in the occurrence and development of cancer. In previous studies, the MTT, CCK-8 cell viability or sulforhodamine B (SRB) assay has been used to measure the cell viability after Huaier treatment $(16,17,19,20)$. The results showed that W-NTRP (a neutral water-soluble polysaccharide isolated from the fruit bodies of Huaier) dose-dependently inhibited the proliferation of three human cholangiocarcinoma cell lines (QBC939, Sk-ChA-1 and MZ-ChA-1) in vitro (16). The same trend was also found in Huaier polysaccharide (HP)-treated hepatocarcinoma cells (MHCC97-H) (21), Huaier extract-treated melanoma cells (A875) (20), ovarian epithelial cancer cells (SKOV3, SKOV3. ip1 and HEY) (17) and breast cancer cells (MCF-7 and MDA-MB-231) (19). The underlying mechanism associated with the proliferation inhibitory effect of Huaier was further revealed in which several well-studied proteins and classic pathways were involved (Fig. 1).

The p53 tumor-suppressor gene can influence cell cycle progression, DNA damage repair, genomic stability and thus inhibit the proliferation of cancer cells (22). To determine whether the proliferation inhibitory effect of Huaier is due to p53-induced cell-cycle arrest, cell cycle distribution of Huaiertreated MCF-7, MDA-MB-231 and A875 cells was analyzed by flow cytometry $(19,20)$. G0/G1 arrest was found in the MCF-7 and MDA-MB-231 cells, suggesting that Huaier inhibits cell proliferation via cell-cycle arrest at the G0/G1 phase (19). The time- and dose-dependent increase in the fraction of cells in the
G2/M phase and the decrease in PCNA protein (a molecular marker for proliferation) expression in the A875 cells treated with Huaier extract confirmed that Huaier extract inhibited melanoma A875 cell proliferation via cell-cycle arrest at the $\mathrm{G} 2 / \mathrm{M}$ phase (20). The enhanced $\mathrm{p} 53$ expression detected in the MCF-7, MDA-MB-231 and A875 cells led to the conclusion that Huaier inhibits cell proliferation via p53-induced cellcycle arrest $(19,20)$.

The $p A K T / m T O R / S 6$ pathway. It has been demonstrated that the AKT/mTOR (protein kinase B/mammalian target of rapamycin) signaling pathway is important for cell growth (23). Ribosomal S6 kinase, a key target of mTOR, controls protein synthesis in cells. Reduced S6 kinase activity leads to a decrease in protein synthesis and inhibits cell growth (24). To detect whether Huaier inhibits proliferation though the pAKT/mTOR/S6 pathway, the protein levels of pAKT, AKT, pS6 (S235-236) and pS6 (S240-244) were evaluated by western blot analysis. The results showed that phosphorylation of S6 at S235-236 and S240-244 was significantly downregulated in three ovarian epithelial cancer cell lines (SKOV3, SKOV3.ip1 and HEY) after $72 \mathrm{~h}$ of Huaier treatment, in accordance with the decreased pAKT expression in SKOV3 and SKOV3.ip1 cells, indicating that the pAKT/ mTOR/S6 kinase pathway is involved in the anti-proliferative effects of Huaier (17).

The ER/NF- $\kappa B$ pathway. Estrogen receptors (ER- $\alpha$ and ER- $\beta$ ) are members of the superfamily of nuclear steroid hormone receptors (25). They regulate the expression of target genes through their binding to specific DNA target sequences or by interacting with other transcription factors (26). A recent study found that Huaier extract efficiently inhibited estrogen- 


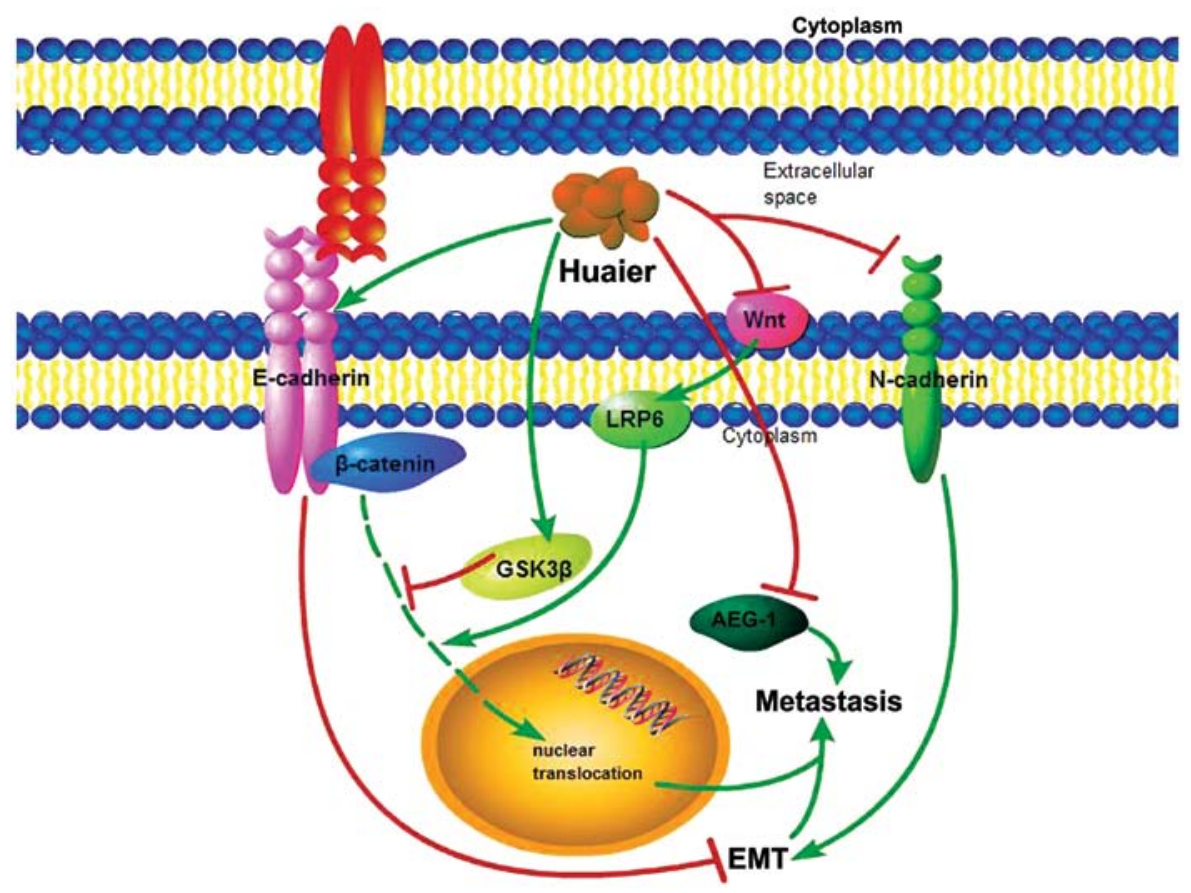

Figure 2. Schema of the underlying mechanism of the anti-metastatic effect of Huaier. Huaier reduces cell mobility in ovarian cancer cells via the GSK3 $\beta /$ $\beta$-catenin signaling pathway. Huaier represses the protein expression and nuclear translocation of $\beta$-catenin due to inhibition of GSK3 $\beta$ S9 phosphorylation and increased LRP6 expression, leading to increased E-cadherin expression and thus inhibition of cell mobility. Inactivation of EMT and the AEG-1 pathway were also found to be involved in the anti-metastatic effect of Huaier in the human hepatocellular carcinoma cell line MHCC97-H, characterized by reduced AEG-1, N-cadherin expression and enhanced E-cadherin expression. EMT, epithelial-mesenchymal transition.

stimulated proliferation in three ER $\alpha$-positive breast cancer cell lines (MCF-7, T47D and ZR-75-1), confirmed by marked downregulation of ER $\alpha$ mRNA and protein levels in Huaiertreated cells. This process was associated with activation of the proteasome (27). The nuclear factor $\kappa \mathrm{B}(\mathrm{NF}-\kappa \mathrm{B})$ pathway, an essential pathway for tumorigenesis (28), is involved in this process. Following Huaier treatment, phosphorylated levels of p65 were reduced almost to the basal level without estrogen, suggesting that Huaier extract abolished the effect of estrogen on the activation of $\mathrm{NF}-\kappa \mathrm{B}$ and thus suppressed the proliferation of breast cancer cells induced by estrogen (27).

The Wnt/ $\beta$-catenin pathway. The classic Wnt/ $\beta$-catenin signaling pathway is essential to human beings since aberrant Wnt/ $\beta$ catenin signaling leads to a series of human diseases including various types of cancers (29). It was demonstrated that Huaier inhibits the proliferation of cancer cells via the $\mathrm{Wnt} / \beta$-catenin signaling pathway (17). The detection of decreased Wnt target gene expression after Huaier treatment provides evidence in support of Wnt/ $\beta$-catenin pathway inhibition. A significant reduction in the expression of DIXDC1 and LRP6 was detected upon Huaier treatment (17). DIXDC1, the human homolog of Ccd1, is a positive Wnt signaling pathway protein that functions downstream of Wnt and upstream of axin and promotes cancer cell proliferation by targeting p21 and cyclin D1 both of which facilitate cell cycle progression from G1 to S-phase (30). LRP6 recruits axin and Dishevelled to the plasma membrane, thereby disrupting the degradation of $\beta$-catenin and facilitating $\beta$-catenin nuclear translocation (31). The downregulation of both DIXDC1 and LRP6 confirmed the anti-proliferative effect of Huaier via the Wnt/ $\beta$-catenin pathway.

\section{Anti-metastatic effect of Huaier}

As one of the major causes of cancer treatment failure and ultimately mortality in cancer patients (32), metastasis is an extremely complicated multi-step process involving the separation of cancer cells from their primary site by penetrating the stromal tissue, circulation through the blood vessels or the lymph nodes, adhesion to the basement membrane and invasion of the target organ for distinct metastasis (33), thereby causing poor prognosis and survival of patients. Moreover, over $90 \%$ of cancer-related deaths are due to metastatic disease (34). Recent studies have shown that Huaier extract suppresses metastasis in ovarian cancer SKOV3, SKOV3.ip1 and HEY cell lines and in the human breast cancer MCF-7 and MDA-MB-231 cell lines, as well as in hepatocarcinoma MHCC97-H cells $(17,19,21)$, indicating that Huaier may serve as a potent anti-metastatic agent for cancer therapy. Migration and scratch assays in vitro were performed to determine the cell migratory ability. In comparison with untreated cells, significant inhibition of migrational movement was observed in migration assays in MDA-MB-231 and MCF-7 cells treated with $4 \mathrm{mg} / \mathrm{ml}$ Huaier (19). In scratch assays, the migration index (corresponding to wound-healing capacity) was significantly inhibited in Huaier-treated SKOV3, SKOV3. ip1 and HEY cells compared with untreated cells, respectively (17). HP exerted the same effect in the scratch assays. The cell migration of human hepatocellular carcinoma cell line MHCC97-H was controlled in a time-dependent manner by HP, being inhibited by up to $28.57,47.81$ and $57.19 \%$ at 25,50 and $100 \mu \mathrm{g} / \mathrm{ml}$, respectively (21). Invasion assays were conducted to compare the invasive potential of Huaier extract- 
treated cells including MDA-MB-231, SKOV3, SKOV3. ip1, HEY and HP-treated MHCC97-H cells with untreated cells. The results revealed that following Huaier treatment the numbers of invading cells through the Matrigel-coated membrane were significantly decreased compared with the untreated groups $(17,19,21)$. In cell adhesion assays performed on MHCC97-H cells, the numbers of adhesive cells on Matrigel-coated plates were stained with hematoxylin and eosin (H\&E) reagent and counted under an inverted microscope. HP treatment significantly inhibited cell adhesion to the Matrigel-coated substrate in a dose-dependent manner; the reduction was $28.55,47.48$ and $56.47 \%$ with $\mathrm{HP}$ at 25,50 and $100 \mu \mathrm{g} / \mathrm{ml}$, respectively (21). The underlying mechanisms of these effects were revealed in several studies suggesting that the GSK3 $\beta / \beta$-catenin and AEG-1 pathways were involved (17,19,21) (Fig. 2). These previous results suggest that Huaier may serve as a potent anti-metastatic agent of enormous clinical value in cancer therapy.

The GSK3 $\beta / \beta$-catenin pathway. In the E-cadherin-mediated cell-cell adhesion cascade, $\beta$-catenin is a key component which can be inhibited by glycogen synthase kinase $3 \beta$ (GSK $3 \beta$ ) via phosphorylation of $\beta$-catenin leading to its ubiquitination and proteasomal degradation $(35,36)$. Yan et al found that following treatment with Huaier aqueous extract for increasing time periods (24, 48 and $72 \mathrm{~h}$ ) and concentrations $(5.0$ and $7.5 \mathrm{mg} / \mathrm{ml}$ ), the cytosolic accumulation and nuclear expression of $\beta$-catenin were markedly decreased in a time-dependent manner in SKOV3, SKOV3. ip1 and HEY cells. Huaier treatment also markedly enhanced total GSK $3 \beta$ expression in a dose-dependent manner in these cell lines while inhibiting GSK3 $\beta$ S9 phosphorylation. The expression of LRP6, a promoter of $\beta$-catenin nuclear translocation, was significantly decreased in the Huaier-treated SKOV3 and SKOV3.ip1 cells. These data indicate that Huaier suppresses not only protein expression, but also the nuclear translocation of $\beta$-catenin due to GSK3 $\beta$ and LRP6. Increased E-cadherin expression was found in the Huaiertreated HEY cells, which inhibits the invasive ability of carcinoma cells. These studies revealed that Huaier inhibited the cell mobility of ovarian cancer cells via the GSK $3 \beta / \beta$ catenin signaling pathway (17).

Inactivation of epithelial-mesenchymal transition (EMT) and the AEG-1 pathway. HP was obtained as a water-soluble brown powder from Huaier extract and was found to exert an antimetastatic effect on human hepatocellular carcinoma cell line MHCC97-H (21). Following wound-healing, cell adhesion and cell invasion assays on MHCC97-H cells treated with various concentrations of $\mathrm{HP}(0,25,50$ or $100 \mu \mathrm{g} / \mathrm{ml})$, it was demonstrated that HP inhibited cell adhesion, invasion and migration in vitro. AEG-1, which has emerged as a vital oncogene in multiple aspects of the development and progression of cancers including HCC (37-39), was significantly decreased after HP treatment. The expression of E-cadherin and N-cadherin as markers of epithelial-mesenchymal transition (EMT) was also detected by western blot analysis. Reduced N-cadherin expression and enhanced E-cadherin expression indicate that the anti-metastatic effect of HP is through inactivation of EMT and the AEG-1 pathway (21).

\section{Anti-angiogenic effect of Huaier}

Angiogenesis is a complex process which is defined as the formation of new blood vessels from pre-existing ones. It involves a multi-step process which includes degradation of the extracellular matrix, migration, proliferation, sprouting, elongation and tube formation of endothelial cells $(40,41)$. Angiogenesis plays an essential role in tumor growth, metastasis and recurrence (42-45). Without vascularization, solid tumors grow only to $1-2 \mathrm{~mm}$ (46). Thus, preventing angiogenesis is a potential strategy for cancer therapy and many anti-angiogenic substances have entered the clinic and have been used as therapeutic options for various types of cancer (47).

Wang et al (14) reported that exposure to Huaier aqueous extract led to cell-cycle arrest, decreased mobility and reduced angiogenesis ability of human umbilical vein endothelial cells (HUVECs), indicating that Huaier may inhibit tumorinduced angiogenesis. The study showed that Huaier increased the proportion of HUVECs in the G0/G1 phase in a dosedependent manner (from $36.79 \pm 2.25 \%$ in the control group to $62.41 \pm 9.77 \%$ in the $8 \mathrm{mg} / \mathrm{ml}$ Huaier group) and promoted the accumulation of p21, a well-studied cyclin-dependent kinase inhibitor, thus resulting in cell-cycle arrest. The motility of HUVECs was then examined using modified scratch and cell migration assays, both of which showed that the motility of the HUVECs was dose- and time-dependently inhibited $(\mathrm{P}<0.01)$. To further evaluate the effect of Huaier on angiogenesis, tube formation, chick embryo chorioallantoic membrane (CAM) and aortic ring assays were subsequently performed in vitro and ex vivo with direct Huaier treatment. The assays revealed that Huaier extract caused a marked decrease in the angiogenesis ability of HUVECs (14). The potential signaling pathways underlying the potent anti-angiogenic activity of Huaier extract were also uncovered. Vascular endothelial growth factor (VEGF), one of the most potent pro-angiogenic factors both physiological and pathological, is a highly specific mitogen for vascular endothelial cells and a vascular permeability enhancer which is essential for endothelial cell proliferation, migration and anti-apoptosis $(48,49)$. Studies have demonstrated that the overexpression of VEGF in cancer patients is associated with poor prognosis and decreased survival $(50,51)$. Huaier extract inhibited the expression of VEGF and the activation of ERK in a dose-dependent manner and eventually exhibited anti-angiogenic activity. Huaier extract also suppressd the phosphorylation of JNK, STAT3 and p65 (major components in the NF- $\kappa \mathrm{B}$ complex), all of which are important pathways that regulate cell migration.

The anti-angiogenic activity in vivo was evaluated in BALB/c mice (14). Tumor tissues from the mice injected with 4T1 cells were stained with H\&E, CD34 and TUNEL. Significantly reduced blood vessels and enhanced apoptosis were observed in mice administered a $100-\mu 1$ solution containing $50 \mathrm{mg}$ Huaier extract by gavage daily. The same effect was also found in tumor-bearing New Zealand rabbits (52). Lower expression of VEGF and P53 and significantly decreased microvessel density (MVD) were observed in the rabbits following treatment with Huaier. These results suggest that Huaier extract may serve as a potent anti-angiogenic and anticancer agent with broad therapeutic value. 


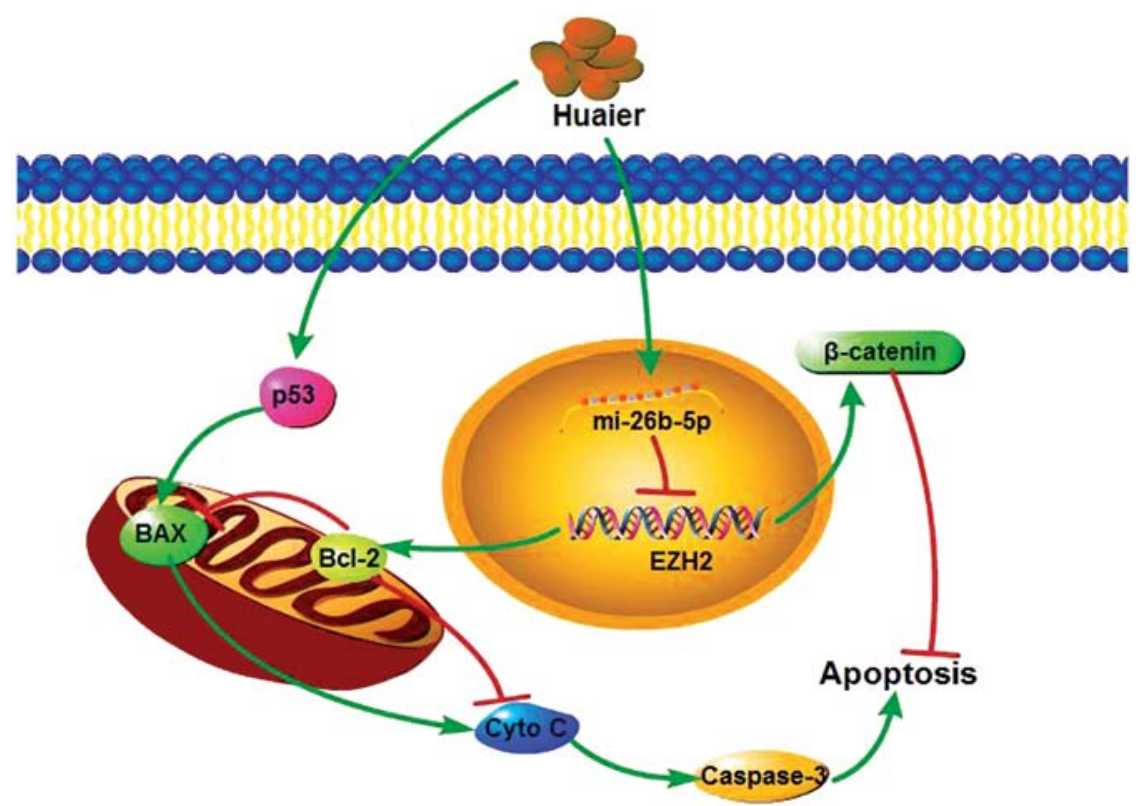

Figure 3. A schema of mechanism of Huaier induced cell apoptosis in a variety of cancer cell lines. Huaier decreased ratio of Bcl-2 to BAX by increasing p53 expression, suggesting the mitochondrial pathway in Huaier induced apoptosis. Huaier could also induce apoptosis via a miR-26b-5p-EZH2-mediated activation of Wnt/ $/$-catenin pathway. EZH2, a protein which was suppressed by miR-26b-5p, could ultimately enhance Bcl-2 expression via the Wnt/ $\beta$-catenin signaling and led to cell apoptosis.

\section{Apoptosis induction by Huaier}

Apoptosis is a genetically encoded program of cell suicide, characterized by the morphological features observed upon cell death which include nuclear condensation, nuclear and cellular fragmentation, membrane blebbing and phagocytosis of the dying cell in the absence of inflammation (53). It is a process that plays an indispensable role in the control of the growth and development of organisms, and serves as a natural barrier to cancer development (54). Blockage of apoptosis may determine the sensitivity of cancer cells to a wide range of diverse chemotherapy agents and explain the frequently observed phenomenon of multidrug resistance. Thus, apoptosis induction is considered to be a crucial strategy for cancer prevention and treatment since successful eradication of cancer cells through apoptosis is one of the ultimate aims of chemotherapy (55). Morphological changes have been observed in cancer cells exposed to Huaier extract. The majority of Huaier-treated melanoma A875 and breast cancer MCF-7 cells became enlarged, irregular-shaped and showed vacuolated changes in the cytoplasm $(19,20)$. These morphological changes demonstrated cell damage following treatment with Huaier extract. Experiments in vitro showed that Huaier inhibited the proliferation of melanoma (A875), lung adenocarcinoma (A549), breast cancer (MCF-7 and MDA-MB-231), hepatocellular carcinoma (Hep-G2) and ovarian cancer (SKOV3, SKOV3.ip1 and HEY) cells by inducing apoptosis $(15,17,19,20,52)$. These previous studies have confirmed that Huaier induces the apoptosis of cancer cells, suggesting that Huaier is an effective anticancer agent for the treatment of cancer patients.

Mitochondria are the targets of several molecular pro-apoptotic signal transduction pathways (56). Intrinsic control of apoptosis requires activation of cytosolic caspases by mitochondrial cytochrome $c$ release and involves regulation of mitochondrial outer membrane permeabilization by Bcl-2 family proteins (57). Mitochondrial-mediated apoptosis has been studied in breast cancer MCF-7, melanoma A875, HCC Hep-G2 and ovarian epithelial cancer SKOV3.ip1 cell lines $(17,19,20,52)$. Following PI/Annexin V double staining performed in MCF-7, A875 and SKOV3.ip1 cells treated with Huaier extract, apoptosis was induced as confirmed by increased late apoptosis or cell death rate (UR) and the early apoptosis rate (LR). Decreased MMP also confirmed Huaier-induced apoptosis in MCF-7 cells (19). Subsequent western blot analysis suggested that this effect was at least partly induced via the mitochondrial pathway. After treatment with Huaier aqueous extract for increasing time periods (48 and $72 \mathrm{~h}$ ) and concentrations ( 4 and $8 \mathrm{mg} / \mathrm{ml}$ ), the expression of p53, phosphorylated-p53 (p-p53), bcl-2 and BAX were tested with western blot analysis. The expression of p53 and p-p53 were upregulated, indicating the accumulation and activation of p53 in response to Huaier treatment in the breast cancer cell line MCF-7. Meanwhile, treatment with Huaier extract suppressed $\mathrm{Bcl}-2$ expression and upregulated BAX expression in a time- and dose-dependent manner, suggesting mitochondrial-mediated apoptosis (19). The same trend was also found in melanoma A875 and ovarian cancer SKOV3.ip1 cells $(17,20)$. In addition, caspase- 3 is the most studied caspase concerning apoptosis induced by natural products (58). It has been reported in previous studies that the activation of caspase-3 was significantly increased in A875, MCF-7 and MDA-MB-231 cells following Huaier treatment, confirmed by increased cleaved caspase- 3 expression and decreased pro-caspase-3 expression $(19,20)$. As detected by immunohistochemistry, Huaier-treated tumor-bearing New Zealand rabbits had lower expression of P53 and Bcl-2 and higher expression of Bax with the differences being significant when compared 
with the control group (52). The results revealed that Huaier extract promoted cell apoptosis through the mitochondrial pathway, thus inhibiting tumor occurrence and progression.

MicroRNAs may act as oncogenes or tumor suppressors involved in the tumorigenesis and aggression of human cancers (59). It was observed in a recent study that Huaier treatment $(2-8 \mathrm{mg} / \mathrm{ml}$ for 24-72 h) induced cell apoptosis of pulmonary adenocarcinoma A549 cells via upregulation of miR-26b-5p (15), an miRNA known to be significantly downregulated in various types of cancer and to induce apoptosis in cancer cells $(60,61)$. Flow cytometric analysis showed that cells treated with $4 \mathrm{mg} / \mathrm{ml}$ Huaier and an miR-26b-5p inhibitor exhibited a $50 \%$ decrease in the rate of apoptosis as compared with the group treated with Huaier and the inhibitor control, indicating that Huaier induced apoptosis via miR-26b-5p. $\mathrm{EZH} 2$, a target gene of miR-26b-5p, is involved in the apoptosis of cancer cells and was found to be downregulated in A549 cells exposed to Huaier. EZH2 is the histone H3 lysine 27 methyltransferase of polycomb-repressive complex 2 and is overexpressed in multiple cancer types (62). Several EZH2-related proteins were detected by western blot analysis in both A549 and non-small cell lung cancer (NSCLC) H1299 cell lines and decreased expression of both $\beta$-catenin and bcl-2 was found in consensus with the previous finding that $\mathrm{EZH} 2$ can ultimately enhance Bcl-2 expression via the activation of Wnt/ $\beta$-catenin signaling (63). Thus, Huaier may induce apoptosis in lung cancer cells via the miR-26b-5p-EZH2-mediated Wnt/ $\beta$-catenin pathway (Fig. 3).

\section{Inhibition of cancer stem cells by Huaier}

Cancer stem cells (CSCs) represent a small subset of cancer-initiating cells endowed with self-renewal and multi-lineage differentiation capacity that promote tumor growth and recurrence (64-66). Clinically, CSCs resist conventional cancer therapies including chemotherapy and radiation therapy and are involved in relapse which is one of the most important features leading to the poor clinical outcome of cancer patients (67-69). Therefore, major clinical challenges towards the complete eradication of cancers are likely to target CSCs (70). In the past few years, the potential role of naturally occurring agents as potent antitumor agents functioning by targeting CSCs has been highlighted (71).

Huaier extract inhibited the number and the size of spheroids formed in both colorectal cancer cells and mammospheres of breast cancer MCF7 cells at a significantly lower concentration than those exerting anti-proliferative impact on bulk cancer cells, indicating that CSCs were preferentially targeted by Huaier extract $(72,73)$. Considering that CD44, CD24 and ALDH are cell surface markers for breast cancer MCF7 and colorectal cancer (CRC) cells, respectively, a decrease in the cell population of $\mathrm{CD} 446^{+} / \mathrm{CD} 24^{-}$breast cancer cells and ALDH-positive CRC cells suggested an inhibitory effect of Huaier on CSCs.

The underlying mechanism of the inhibitory effect of Huaier on CSCs was subsequently studied, among which downregulation of the $\mathrm{Wnt} / \beta$-catenin pathway and inhibition of the hedgehog (Hh) pathway were highlighted (Fig. 4). The Wnt/ $\beta$-catenin pathway is one of the critical pathways demonstrated to mediate the self-renewal of CSCs (74). The activation of Wnt target genes depends on mediation by $\beta$-catenin, which enters the nucleus to activate the TCF/LEF transcription factor (74). Treatment of the CRC cells with Huaier led to dose-dependent downregulation of the levels of total $\beta$-catenin protein, decreased activation of TCF/LEF in the nucleus and reduced expression of cyclin D1, one of the Wnt $/ \beta$-catenin downstream genes (72). Therefore, the downregulation of the $\mathrm{Wnt} / \beta$-catenin self-renewal pathway may be a potential target of the Huaier extract. The inhibitory effect of Huaier extract on CSCs was also found to be partly dependent on the inactivation of the Hh pathway which is associated with both normal mammary gland development and breast cancer progression $(73,75)$. After treatment with Huaier extract, the expression of Gli1 was obviously declined, showing that Huaier extract was highly effective to eradicate breast (CSCs) through regulating Hh signaling (73). These results identified Huaier as an effective agent of great clinical value to eradicate CSCs and to improve current cancer treatment.

\section{Tumor-specific immunomodulatory effect of Huaier}

Tumor growth and the immune system are intertwined in a complex competition where tilting the subtle balance between tumor-specific immunity and tolerance can ultimately decide the fate of the host (76). Recently, experimental and clinical testing of novel types of immunotherapeutic agents have been rapidly developing due to the advances in tumor immunology and a better understanding of the mechanisms regulating the immune response (77). The water extract from Huaier consists mainly of polysaccharide protein which has been proved to be of great clinical value as the main active ingredient in the anticancer effects and immunity-enhancing actions of Huaier (Fig. 5) (16). Lymphocytes are the key effector cells of the mammalian immune system, whose proliferation is considered an indicator of immunopotentiation. W-NTRP (a neutral water-soluble polysaccharide isolated from the fruit bodies of Huaier) activated different subpopulations of lymphocytes and was suggested to be a potent immunomodulating and immunoenhancing agent (16). TP-1 (a Huaier polysaccharide) administered at two doses (50 or $100 \mathrm{mg} / \mathrm{kg}$ ) significantly prompted the T- or B-lymphocyte proliferation induced by ConA or LPS, respectively, when compared to those in H22-bearing mice (Kunming mice weekly transplanted with murine $\mathrm{H} 22$ ascitic hepatoma cells into the peritoneal cavities). Li et al (78) also noted increased $\mathrm{CD}^{+}$ $\mathrm{T}$ cells and decreased $\mathrm{CD} 8^{+} \mathrm{T}$ cells induced by TP-1 in tumorbearing mice (78). Macrophages and NK cells are capable of inducing the death of tumor cells and play essential roles in host anticancer immune response (79). Macrophages are an essential component of the host defense against tumor growth by releasing various cell factors, such as TNF- $\alpha$, NO, reactive oxygen intermediates (ROI) and other substances to kill tumor cells (80). It was reported that W-NTRP significantly enhanced macrophage phagocytosis, increasing NO production and iNOS activity (16). An increased quantity of NK cells and enhanced NK cell activity were detected after exposure to Huaier polysaccharide $(18,78)$. Huaier polysaccharide (TP-1) also modulated the cytokine release, as confirmed by the 


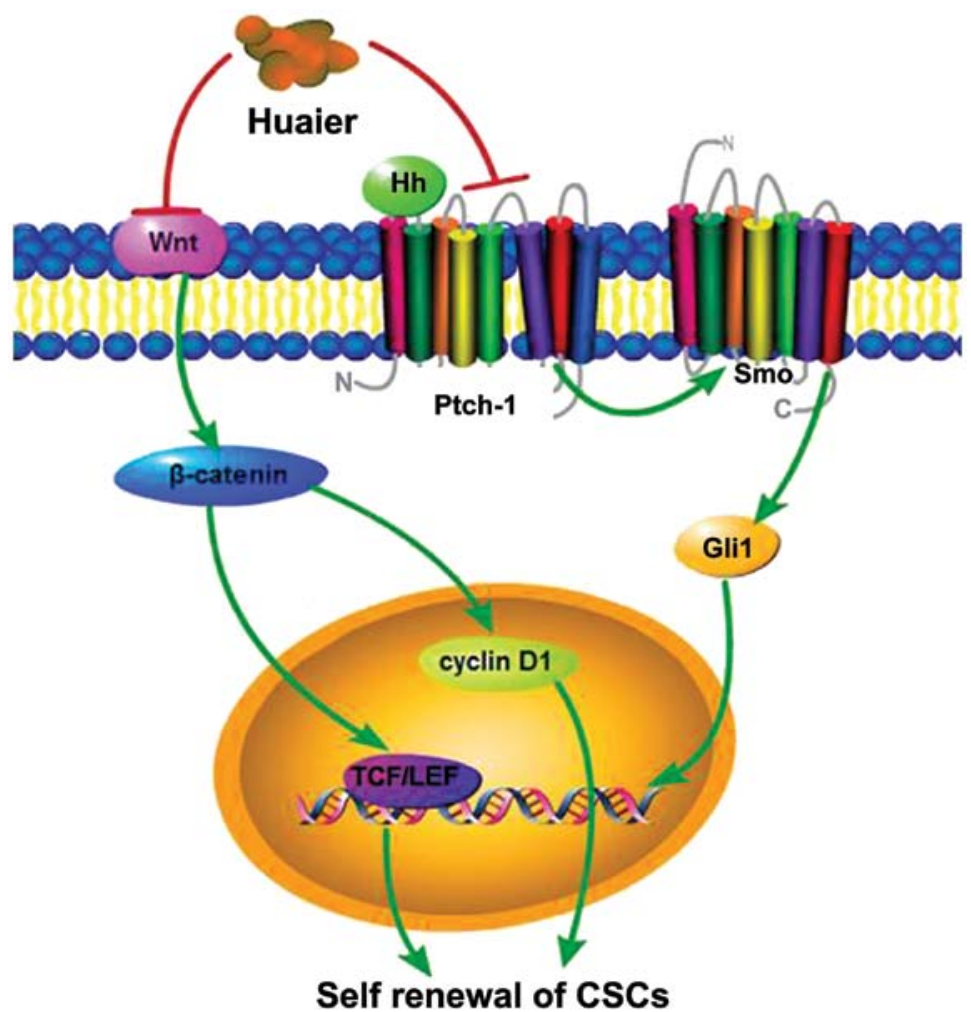

Figure 4. Schematic representation of the inhibitory effect of Huaier on CSCs. The inhibitory effect of Huaier on CSCs is associated with the downregulation of the Wnt/ $\beta$-catenin pathway and inhibition of the hedgehog pathway. Following Huaier treatment, downregulation of the levels of $\beta$-catenin protein, decreased activation of TCF/LEF in the nucleus and cyclin D1 expression suggest that the downregulation of the Wnt/ $\beta$-catenin self-renewal pathway is a potential target of Huaier extract. The decreased expression of Gli1 indicates that Huaier extract is highly effective to eradicate breast cancer stem cells bu regulating Hh signaling. CSCs, cancer stem cells.

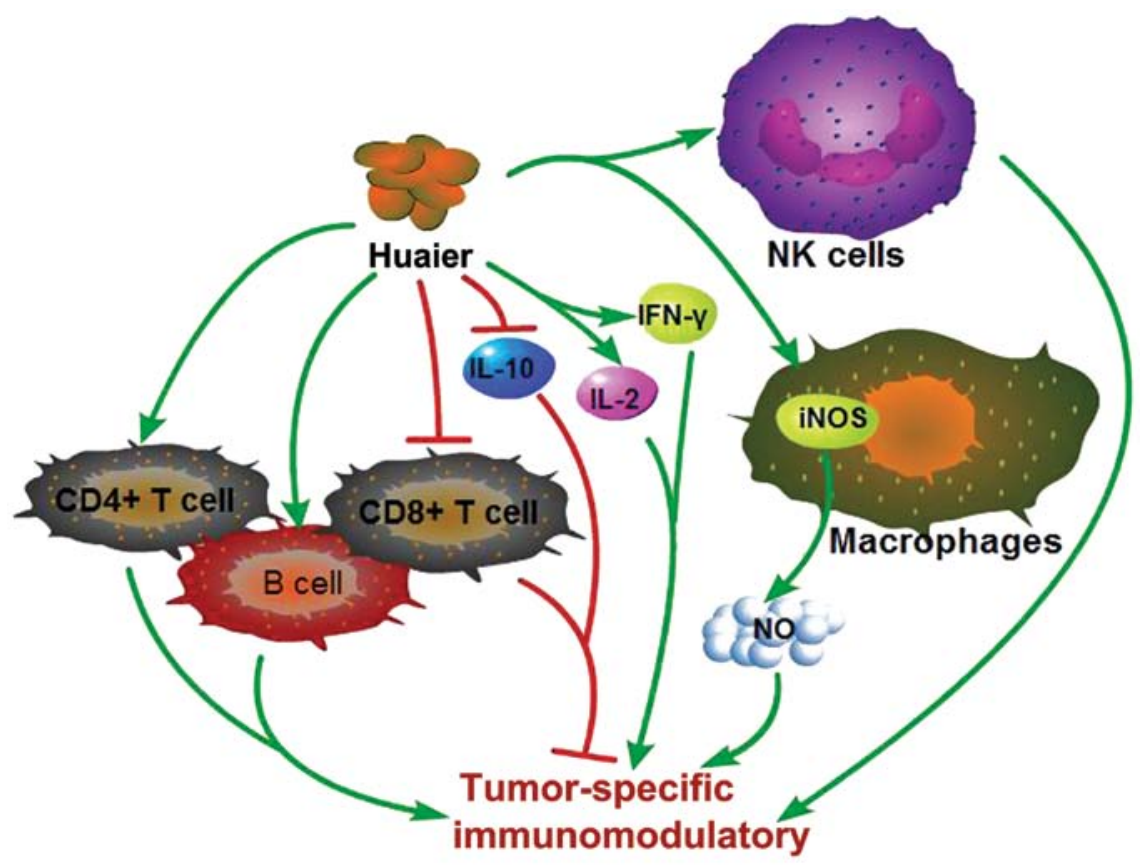

Figure 5. Tumor-specific immunomodulatory effect of Huaier. Huaier significantly activates lymphocytes, prompts the T- or B-lymphocyte proliferation, enhances macrophage phagocytosis and NO production, increases the quantity of NK cells and enhances NK cell activity, modulates cytokine release such as IFN- $\gamma$, IL-2 and IL-10 expression, all of which lead to the tumor-specific immune response induced by Huaier treatment.

increased IFN- $\gamma$ and IL-2 expression and inhibition of IL-10 expression. Taken together, these results suggest that the poly- saccharide isolated from Huaier has an immunoregulatory effect and potent antitumor activities with great clinical value. 


\section{Therapeutic perspectives and conclusions}

Huaier, as a type of officinal fungi, has been used in China for nearly 1,600 years, and has been reported to exert potent anticancer activities. This review aimed at obtaining a clear picture regarding the anticancer effects of Huaier and the underlying mechanisms. The anticancer potential of Huaier, including proliferation inhibitory, anti-metastasis, anti-angiogenesis, apoptosis induction, tumor-specific immunomodulatory and cancer stem cell inhibitory activities, have been validated in in vitro studies and in various animal models, indicating its potential therapeutic value against various types of cancers. Another encouraging finding was that Huaier may serve as an efficient anticancer agent with little toxicity. Preliminary toxicological evaluation demonstrated that TP-1 (a Huaier polysaccharide) had no obvious systemic toxicity on the kidney and liver of tumor-bearing mice at the therapeutic dose ( 25 and $50 \mathrm{mg} / \mathrm{kg}$ ). This was confirmed by the results that the tumor-caused changes in hepatic function markers (ALT and AST) and renal function parameters (BUN, UA, and CRE) (78) were ameliorated, or even restored to the normal level after TP-1 treatment. Xenograft experiments in mice were also performed. The weight of tumors isolated from the Huaier-treated groups (at concentration of $2.5 \mathrm{~g} / \mathrm{kg}$ /day) was significantly decreased compared to the control group with no significant difference in body weight, which indicated no obvious toxicity to mice at the curative dose (17). Several molecular targets and classic pathways of Huaier which play an important role in the development of cancer have also been identified. These results highlight the possible application of Huaier in cancer chemoprevention and lay a solid foundation for its clinical use in humans, opening a more effective anticancer treatment option even though most of the research is still in an experimental stage. We hope and believe that Huaier may provide a benefit in the treatment of malignant tumors as a prospective anticancer drug candidate.

Although the findings documented in this review are quite encouraging for the use of Huaier as a novel anticancer agent, several limitations exist in the current scenario. Most of the studies were conducted using Huaier aqueous extract, and the observed biological effects could be due to the combined effects of various components. Thus, further research on the specific effective anticancer components of Huaier is warranted. Although animal and experimental studies have been successfully carried out showing Huaier's efficacy as an anticancer agent, no information on the absorption, distribution, metabolism, and excretion of Huaier in humans, or clinical trial was found in the search of the available literature. Therefore, clinical studies should be carried out to illustrate the untapped chemopreventive and therapeutic potential of Huaier either alone or in conjunction with existing therapies.

\section{Acknowledgements}

The present study was supported by the National Natural Science Foundation of China (nos. 81272903, 81172529 and 81072150), and the Shandong Science and Technology Development Plan (nos. 2012GZC22115 and 2013GRC31801) to Q.Y.

\section{References}

1. Winter-Roach B, Hooper L and Kitchener H: Systematic review of adjuvant therapy for early stage (epithelial) ovarian cancer. Int J Gynecol Cancer 13: 395-404, 2003.

2. Wolpin BM, Meyerhardt JA, Mamon HJ and Mayer RJ: Adjuvant treatment of colorectal cancer. CA Cancer J Clin 57: 168-185, 2007.

3. Macdonald JS, Smalley SR, Benedetti J, Hundahl SA, Estes NC, Stemmermann GN, Haller DG, Ajani JA, Gunderson LL, Jessup JM and Martenson JA: Chemoradiotherapy after surgery compared with surgery alone for adenocarcinoma of the stomach or gastroesophageal junction. New Engl J Med 345: 725-730, 2001.

4. Liao WC, Chien KL, Lin YL Wu MS, Lin JT, Wang HP and Tu YK: Adjuvant treatments for resected pancreatic adenocarcinoma: a systematic review and network meta-analysis. Lancet Oncol 14: 1095-1103, 2013.

5. Wirth T, Parker N and Ylä-Herttuala S: History of gene therapy. Gene 525: 162-169, 2013.

6. Parums DV: Current status of targeted therapy in non-small cell lung cancer. Drugs Today (Barc) 50: 503-525, 2014.

7. Pan MH and Ho CT: Chemopreventive effects of natural dietary compounds on cancer development. Chem Soc Rev 37: 2558-2574, 2008.

8. Kannaiyan R, Shanmugam MK and Sethi G: Molecular targets of celastrol derived from Thunder of God Vine: potential role in the treatment of inflammatory disorders and cancer. Cancer Lett 303: 9-20, 2011.

9. Yu J, Liu H, Lei J, Tan W, Hu X and Zou G: Antitumor activity of chloroform fraction of Scutellaria barbata and its active constituents. Phytotherapy Res 21: 817-822, 2007.

10. Harhaji Lj, Mijatović S, Maksimović-Ivanić D, Stojanović I, Momcilović M, Maksimović V, Tufegdzić S, Marjanović Z, Mostarica-Stojković M, Vucinić Z, et al: Anti-tumor effect of Coriolus versicolor methanol extract against mouse B16 melanoma cells: in vitro and in vivo study. Food Chem Toxicol 46: 1825-1833, 2008

11. Rashid S, Unyayar A, Mazmanci MA, McKeown SR, Banat IM and Worthington J: A study of anti-cancer effects of Funalia trogii in vitro and in vivo. Food Chem Toxicol 49: 1477-1483, 2011.

12. McCulloch M, See C, Shu XJ, Broffman M, Kramer A, Fan WY, Gao J, Lieb W, Shieh K and Colford JM Jr: Astragalus-based Chinese herbs and platinum-based chemotherapy for advanced non-small-cell lung cancer: meta-analysis of randomized trials. J Clin Oncol 24: 419-430, 2006.

13. Li LX, Ye SL, Wang YH and Tang ZZ: Progress on experimental research and clinical application of Trametes robiniophila. Bull Chin Cancer 16: 110-113, 2007.

14. Wang X, Zhang N, Huo Q and Yang Q: Anti-angiogenic and antitumor activities of Huaier aqueous extract. Oncol Rep 28: 1167-1175, 2012.

15. Wu T, Chen W, Liu S, Lu H, Wang H, Kong D, Huang X, Kong Q, Ning Y and Lu Z: Huaier suppresses proliferation and induces apoptosis in human pulmonary cancer cells via upregulation of miR-26b-5p. FEBS Lett 588: 2107-2114, 2014.

16. Sun Y, Sun T, Wang F, Zhang J, Li C, Chen X, Li Q and Sun S: A polysaccharide from the fungi of Huaier exhibits anti-tumor potential and immunomodulatory effects. Carbohydr Polym 92: 577-582, 2013

17. Yan X, Lyu T, Jia N, Yu Y, Hua K and Feng W: Huaier aqueous extract inhibits ovarian cancer cell motility via the AKT/GSK3beta/ beta-catenin pathway. PloS One 8: e63731, 2013.

18. Zheng J, Li C, Wu X, Liu M, Sun X, Yang Y, Hao M, Sheng S, Sun Y,Zhang H, et al: Astrocyte elevated gene-1 (AEG-1) shRNA sensitizes Huaier polysaccharide (HP)-induced anti-metastatic potency via inactivating downstream P13K/Akt pathway as well as augmenting cell-mediated immune response. Tumour Biol 35: 4219-4224, 2014

19. Zhang N, Kong X, Yan S, Yuan C and Yang Q: Huaier aqueous extract inhibits proliferation of breast cancer cells by inducing apoptosis. Cancer Sci 101: 2375-2383, 2010.

20. Zhang F, Zhang Z and Liu Z: Effects of Huaier aqueous extract on proliferation and apoptosis in the melanoma cell line A875. Acta Histochem 115: 705-711, 2013.

21. Zheng J, Li C, Wu X, Liu M, Sun X, Yang Y, Hao M, Sheng S, Sun Y, Zhang H, et al: Huaier polysaccharides suppresses hepatocarcinoma MHCC97-H cell metastasis via inactivation of EMT and AEG-1 pathway. Int J Biol Macromol 64: 106-110, 2014. 
22. Pflaum J, Schlosser S and Müller M: p53 Family and cellular stress responses in cancer. Front Oncol 4: 285, 2014.

23. Advani AS, Mahfouz RZ, Maciejewski J, Rybicki L, Sekeres M, Tripp B, Kalaycio M, Bates J and Saunthararajah Y: Ribosomal S6 kinase and AKT phosphorylation as pharmacodynamic biomarkers in patients with myelodysplastic syndrome treated with RAD001. Clin Lymphoma Myeloma Leuk 14: 172-177, 2014.

24. Magnuson B, Ekim B and Fingar DC: Regulation and function of ribosomal protein S6 kinase (S6K) within mTOR signalling networks. Biochem J 441: 1-21, 2012.

25. Hedden A, Müller V and Jensen EV: A new interpretation of antiestrogen action. Ann NY Acad Sci 761: 109-120, 1995.

26. Klinge CM: Estrogen receptor interaction with estrogen response elements. Nucleic Acids Res 29: 2905-2919, 2001.

27. Wang X, Zhang N, Huo Q, Sun M, Lv S and Yang Q: Huaier aqueous extract suppresses human breast cancer cell proliferation through inhibition of estrogen receptor $\alpha$ signaling. Int J Oncol 43: 321-328, 2013.

28. Shen HM and Tergaonkar V: NFkappaB signaling in carcinogenesis and as a potential molecular target for cancer therapy. Apoptosis 14: 348-363, 2009.

29. Barker $\mathrm{N}$ and Clevers $\mathrm{H}$ : Mining the Wnt pathway for cancer therapeutics. Nat Rev Drug Discov 5: 997-1014, 2006.

30. Wang L, Cao XX, Chen Q, Zhu TF, Zhu HG and Zheng L: DIXDC1 targets $\mathrm{p} 21$ and cyclin D1 via PI3K pathway activation to promote colon cancer cell proliferation. Cancer Sci 100 1801-1808, 2009.

31. MacDonald BT, Tamai K and He X: Wnt/beta-catenin signaling: components, mechanisms, and diseases. Dev Cell 17: 9-26, 2009.

32. Jin X, Zhu Z and Shi Y: Metastasis mechanism and gene/protein expression in gastric cancer with distant organ metastasis. Bull Cancer: Oct 8, 2014 (Epub ahead of print).

33. Song IH: Cancer metastasis and metastasis suppressors. Korean J Gastroenterol 43: 1-7, 2004. (In Korean).

34. Grinshpun A, Ben-Porath I, Peretz T and Salmon A: Tumor, metastasis and what's in between. Harefuah 152: 30-33, 59, 58 , 2013.

35. Bajpai S, Feng Y, Wirtz D and Longmore GD: $\beta$-Catenin serves as a clutch between low and high intercellular E-cadherin bond strengths. Biophys J 105: 2289-2300, 2013.

36. Liu H, Xu GH, Wang K, Cao JL, Gu EW, Li YH and Liu XS Involvement of GSK3 $/ \beta$-catenin signaling in the impairment effect of ketamine on spatial memory consolidation in rats. Neurobiol Learn Mem 111: 26-34, 2014

37. Yuan L, Shi RR, Rao SM, Song JL and Cui MC: Reversal of resistance to adriamycin in human breast cancer cell line MCF-7/ADM by silencing AEG-1 gene and its mechanism. Sheng Li Xue Bao 66: 625-630, 2014 (In Chinese)

38. Ke ZF, Mao X, Zeng C, He S, Li S and Wang LT: AEG-1 expression characteristics in human non-small cell lung cancer and its relationship with apoptosis. Med Oncol 30: 383, 2013.

39. Hu G, Wei Y and Kang Y: The multifaceted role of MTDH/AEG-1 in cancer progression. Clin Cancer Res 15: 5615-5620, 2009.

40. Risau W: Mechanisms of angiogenesis. Nature 386: 671-674, 1997.

41. Carmeliet P and Jain RK: Angiogenesis in cancer and other diseases. Nature 407: 249-257, 2000.

42. Tassi E and Wellstein A: Tumor angiogenesis: initiation and targeting - therapeutic targeting of an FGF-binding protein, an angiogenic switch molecule, and indicator of early stages of gastrointestinal adenocarcinomas. Cancer Res Treat 38: 189-197, 2006.

43. Hanahan D and Folkman J: Patterns and emerging mechanisms of the angiogenic switch during tumorigenesis. Cell 86: 353-364, 1996.

44. Bonapace L, Coissieux MM, Wyckoff J, Mertz KD, Varga Z, Junt T and Bentires-Alj M: Cessation of CCL2 inhibition accelerates breast cancer metastasis by promoting angiogenesis. Nature 515: 130-133, 2014.

45. Phoophitphong T, Hanprasertpong J, Dechsukhum $\mathrm{C}$ and Geater A: Correlation of angiogenesis and recurrence-free survival of early stage cervical cancer patients undergoing radical hysterectomy with pelvic lymph node dissection. J Obstet Gynaecol Res 33: 840-848, 2007.

46. Folkman J: Tumor angiogenesis: therapeutic implications. N Engl J Med 285: 1182-1186, 1971

47. Tang HS, Feng YJ and Yao LQ: Angiogenesis, vasculogenesis, and vasculogenic mimicry in ovarian cancer. Int $\mathrm{J}$ Gynecol Cancer 19: 605-610, 2009.
48. Giuliano S and Pagès G: Mechanisms of resistance to antiangiogenesis therapies. Biochimie 95: 1110-1119, 2013.

49. Neufeld G, Cohen T, Gengrinovitch S and Poltorak Z: Vascula endothelial grow th factor (VEGF) and its receptors. FASEB J 13: 9-22, 1999.

50. Paley PJ, Staskus KA, Gebhard K, Mohanraj D, Twiggs LB, Carson LF and Ramakrishnan S: Vascular endothelial growth factor expression in early stage ovarian carcinoma. Cancer 80 : 98-106, 1997.

51. Chen P, Zhu J, Liu DY, Li HY, Xu N and Hou M: Over-expression of survivin and VEGF in small-cell lung cancer may predict the poorer prognosis. Med Oncol 31: 775, 2014.

52. Ren J, Zheng C, Feng G, Liang H, Xia X, Fang J, Duan X and Zhao H: Inhibitory effect of extract of fungi of Huaier on hepatocellular carcinoma cells. J Huazhong Univ Sci Technolog Med Sci 29: 198-201, 2009

53. Wyllie AH, Kerr JF and Currie AR: Cell death: the significance of apoptosis. Int Rev Cytol 68: 251-306, 1980.

54. Adams JM and Cory S: The Bcl-2 apoptotic switch in cancer development and therapy. Oncogene 26: 1324-1337, 2007.

55. Lowe SW and Lin AW: Apoptosis in cancer. Carcinogenesis 21: 485-495, 2000

56. Green DR, Galluzzi L and Kroemer G: Cell biology. Metabolic control of cell death. Science 345: 1250256, 2014.

57. Degli Esposti M: Mitochondria in apoptosis: past, present and future. Biochem Soc Trans 32: 493-495, 2004.

58. Costa M, Costa-Rodrigues J, Fernandes MH, Barros P, Vasconcelos V and Martins R: Marine cyanobacteria compounds with anticancer properties: a review on the implication of apoptosis. Mar Drugs 10: 2181-2207, 2012.

59. Huang Y, Yang YB, Zhang XH, Yu XL, Wang ZB and Cheng XC: MicroRNA-21 gene and cancer. Med Oncol 30: 376, 2013.

60. Solomides CC, Evans BJ, Navenot JM, Vadigepalli R, Peiper SC and Wang ZX: MicroRNA profiling in lung cancer reveals new molecular markers for diagnosis. Acta Cytol 56: 645-654, 2012.

61. Liu XX, Li XJ, Zhang B, Liang YJ, Zhou CX, Cao DX, He M, Chen GQ, He JR and Zhao Q: MicroRNA-26b is underexpressed in human breast cancer and induces cell apoptosis by targeting SLC7A11. FEBS Lett 585: 1363-1367, 2011.

62. Koh CM, Iwata T, Zheng Q, Bethel C, Yegnasubramanian S and De Marzo AM: Myc enforces overexpression of EZH2 in early prostatic neoplasia via transcriptional and post-transcriptional mechanisms. Oncotarget 2: 669-683, 2011.

63. Yuan JB, Yang LY, Tang ZY, Zu XB and Qi L: Down-regulation of EZH2 by RNA interference inhibits proliferation and invasion of ACHN cells via the Wnt/ $\beta$-catenin pathway. Asian Pac J Cancer Prev 13: 6197-6201, 2012.

64. Reya T, Morrison SJ, Clarke MF and Weissman IL: Stem cells, cancer, and cancer stem cells. Nature 414: 105-111, 2001.

65. Dalerba P, Cho RW and Clarke MF: Cancer stem cells: models and concepts. Annu Rev Med 58: 267-284, 2007.

66. Ricci-Vitiani L, Lombardi DG, Pilozzi E, Biffoni M, Todaro M, Peschle C and De Maria R: Identification and expansion of human colon-cancer-initiating cells. Nature 445: 111-115, 2007.

67. Rycaj K and Tang DG: Cancer stem cells and radioresistance. Int J Radiat Biol 90: 615-621, 2014.

68. Chen K, Huang YH and Chen JL: Understanding and targeting cancer stem cells: therapeutic implications and challenges. Acta Pharmacol Sin 34: 732-740, 2013.

69. Beier D, Schulz JB and Beier CP: Chemoresistance of glioblastoma cancer stem cells--much more complex than expected. Mol Cancer 10: 128, 2011.

70. Tsujii M: Cancer therapy targeting cancer stem cell. Nihon rinsho 72: 35-41, 2014. (In Japanese).

71. Bao B, Li Y, Ahmad A, Azmi AS, Bao G, Ali S, Banerjee S, Kong D and Sarkar FH: Targeting CSC-related miRNAs for cancer therapy by natural agents. Curr Drug Targets 13: $1858-1868,2012$

72. Zhang T1, Wang K, Zhang J, Wang X, Chen Z, Ni C, Qiu F and Huang J: Huaier aqueous extract inhibits colorectal cancer stem cell growth partially via downregulation of the Wnt $/ \beta$-catenin pathway. Oncol Lett 5: 1171-1176, 2013.

73. Wang X, Zhang N, Huo Q, Sun M, Dong L, Zhang Y, Xu G and Yang Q: Huaier aqueous extract inhibits stem-like characteristics of MCF7 breast cancer cells via inactivation of hedgehog pathway. Tumour Biol 35: 10805-10813, 2014

74. Clevers H: Wnt/beta-catenin signaling in development and disease. Cell 127: 469-480, 2006. 
75. Gangopadhyay S, Nandy A, Hor P and Mukhopadhyay A: Breast cancer stem cells: a novel therapeutic target. Clin Breast Cancer 13: 7-15, 2013.

76. Townsend KN, Hughson LR, Schlie K, Poon VI, Westerback A and Lum JJ: Autophagy inhibition in cancer therapy: metabolic considerations for antitumor immunity. Immunol Rev 249: 176-194, 2012.

77. Ladányi A and Balatoni T: Unblocking antitumor immune response: novel possibilities for the immunotherapy of melanoma. Magy Onkol 57: 100-107, 2013. (In Hungarian).

78. Li C, Wu X, Zhang H, Yang G, Hao M, Sheng S, Sun Y, Long J, $\mathrm{Hu}$ C, Sun X, et al: A Huaier polysaccharide inhibits hepatocellular carcinoma growth and metastasis. Tumour Biol: Nov 6 , 2014 (Epub ahead of print).
79. Stuelp-Campelo PM, de Oliveira MB, Leão AM, Carbonero ER, Gorin PA and Iacomini M: Effect of a soluble alpha-D-glucan from the lichenized fungus Ramalina celastri on macrophage activity. Int Immunopharmacol 2: 691-698, 2002.

80. Fidler IJ and Kleinerman ES: Therapy of cancer metastasis by systemic activation of macrophages: from the bench to the clinic. Res Immunol 144: 284-287, 1993. 